\title{
Multicast Capacity for Hybrid Wireless Networks
}

\author{
XuFei Mao \\ Dept. of Computer Science \\ Illinois Institute of Technology \\ Chicago, IL 60616, USA \\ xmao3@iit.edu
}

\author{
Xiang-Yang Li * \\ Dept. of Computer Science \\ Illinois Institute of Technology \\ Chicago, IL, USA \\ xli@cs.iit.edu
}

\author{
ShaoJie Tang \\ Dept. of Computer Science \\ Illinois Institute of Technology \\ Chicago, IL 60616, USA \\ stang7@iit.edu
}

\begin{abstract}
We study the multicast capacity of a random wireless network consisting of ordinary wireless nodes and base stations, known as a hybrid network. Assume that $n$ ordinary wireless nodes are randomly deployed in a square region and all nodes have the uniform transmission range $r$ and uniform interference range $R>r$. We further assume that each ordinary wireless node can transmit/receive at $W$ bits/second over a common wireless channel. In addition, there are $m$ additional base stations (neither source nodes nor receiver nodes) placed regularly in this square region and connected by a high-bandwidth wired network. For each ordinary node $v$, we randomly pick $k-1$ nodes from the other $n-1$ ordinary nodes as the receivers of the multicast session rooted at node $v$. The aggregated multicast capacity is defined as the total data rate of all multicast sessions in this hybrid network.

We derive asymptotic upper bounds and lower bounds on multicast capacity of the hybrid wireless networks. The total multicast capacity is $O\left(\frac{\sqrt{n}}{\sqrt{\log n}} \cdot \frac{\sqrt{m}}{k} \cdot W\right)$ when $k=O\left(\frac{n}{\log n}\right), k=$ $O(m), \frac{k}{\sqrt{m}} \rightarrow \infty$ and $m=o\left(\frac{a^{2}}{r^{2}}\right)$; the total multicast capacity is $\Theta\left(\frac{\sqrt{n}}{\sqrt{\log n}} \cdot \frac{W}{\sqrt{k}}\right)$ when $k=O\left(\frac{n}{\log n}\right), k=\Omega(m)$ and $\frac{m}{k} \rightarrow 0$. When $k=O\left(\frac{n}{\log n}\right)$ and $k=O(\sqrt{m})$, the upper bound for the minimum multicast capacity is at most $O\left(\frac{r \cdot n}{a} \cdot \sqrt{m} \cdot \frac{W}{k}\right)$ and is at least $\Omega(W)$ respectively. When $k=\Omega\left(\frac{n}{\log n}\right)$, the multicast capacity is $\Theta(W)$.
\end{abstract}

\section{Categories and Subject Descriptors}

C.2.1 [Network Architecture and Design]: Wireless communication, Network topology; G.2.2 [Graph Theory]: Network problems, Graph algorithms

${ }^{*}$ The research of the author was partially supported by National Basic Research Program of China (973 Program) under grant No. 2006CB30300, the National High Technology Research and Development Program of China (863 Program) under grant No. 2007AA01Z180, the RGC under Grant HKBU 2104/06E and CERG under Grant PolyU-5232/07E. Partial of the work was done when the author visited Microsoft Research Asia, and State Key Laboratory of Novel Software Technology, NanJing University.

Permission to make digital or hard copies of all or part of this work for personal or classroom use is granted without fee provided that copies are not made or distributed for profit or commercial advantage and that copies bear this notice and the full citation on the first page. To copy otherwise, to republish, to post on servers or to redistribute to lists, requires prior specific permission and/or a fee.

MobiHoc'08, May 26-30, 2008, Hong Kong SAR, China.

Copyright 2008 ACM 978-1-60558-083-9/08/05 ...\$5.00.

\section{General Terms}

Algorithms, Design, Theory

\section{Keywords}

Hybrid wireless networks, capacity, multicast, broadcast.

\section{INTRODUCTION}

The capacity has been widely studied for different types of wireless networks, like ad hoc wireless networks, cellular networks, etc. The main purpose of this paper is to study the multicast capacity of hybrid wireless networks when we choose the best protocols for all layers. A hybrid wireless network consists of two types of network terminals: base stations and wireless nodes. We assume that all base stations are equipped with wireless network interface cards such that they can communicate with wireless nodes nearby; We further assume that a base station is neither a data source nor a data receiver, it simply serves as a relaying communication gateway.

Basically, there are two different routing strategies in the hybrid wireless network. The first case is that when a source node and some of its receiver nodes fall in the same subregion, the source node will try to reach these receivers by the multicast tree (may need some relay nodes) inside the subregion. Otherwise, the source node will try to reach the closest base station first through one- or multi-hop, and then the latter will relay the data to other base stations which are closest to those receivers outside the subregion. At last, each of these base stations carrying the data will act as a root of a multicast tree to relay the data to receivers by one- or multihop (may need other relaying wireless nodes). We simply call this routing strategy as pure hybrid routing. On the other hand, with the increasing number of source nodes inside one subregion, if most of source nodes have some receivers outside the subregion, the base stations may have much burden to relay data, thus become bottlenecks. In this case, the wireless source nodes switch to use globally multicast trees to send data to their receivers rather than using base stations. This approach has the same capacity as the ad-hoc wireless network. We call this routing strategy as pure ad hoc routing. Thus, a hybrid wireless network actually presents a tradeoff by combining a traditional BS-oriented network with a pure ad hoc wireless network.

As in the literature, we mainly consider several types of networks based on the node density and deployment patterns of the base stations. We study the capacity of a given hybrid wireless network where the nodes' positions are given a priori, and how the capacity of a hybrid wireless network scales with the number of nodes or with the number of base stations in the network when given a fixed deployment region, or scales with the size of the deployment region for multicast when given a fixed deployment density. We assume 
that a set of $n$ wireless nodes are randomly distributed in a square region with side length $a$. In addition, a set of $m$ base stations are regularly distributed in this region. For most results presented in this paper, we assume that the deployment region is selected such that the resultant network will be connected with high probability.

The results derived under this model also imply the same results for the dense model, when $n$ nodes are distributed in a fixed region (such as a unit square by a proper scaling) and the uniform transmission range of all nodes is selected as the critical transmission range to get a connected network with high probability (from now on we abbreviate with high probability to w.h.p.).

For all randomly distributed $n$ nodes, each node $v_{i}$ has randomly chosen $k-1$ destination nodes from other $n-1$ wireless nodes, to which it wishes to send data at an arbitrary data rate $\lambda_{i}$. The multicast capacity of a random network is defined as $\sum_{i=1}^{n} \lambda_{i}$ when there is a schedule of transmissions so that all multicast flows will be received by their destination nodes successfully.

We assume that each wireless node can transmit at $W$ bits/second over a common wireless channel. For presentation simplicity, we assume that there is only one channel in the hybrid wireless networks. As always, we assume that a wireless node has enough memory to buffer all the packets it generated or relayed for others such that no packets will be lost through transmission. We leave it as future work to study the scenario in which the buffers of intermediate nodes are bounded by some values. For most of the results presented here, the delay of the routing is not considered, i.e., the delay in the worst case could be arbitrarily large for some results.

Our Main Contributions: In this paper we derive analytical upper bounds and lower bounds on multicast capacity of a hybrid wireless network, in which base stations are distributed regularly in a grid. Assume that the deployment region and the transmission range $r$ are selected ${ }^{1}$ such that the network is connected w.h.p. We show that when base stations are distributed regularly, the aggregated multicast capacity of $n$ multicast sessions is

$$
\Lambda_{k}(n)= \begin{cases}O\left(\frac{\sqrt{n}}{\sqrt{\log n}} \cdot \frac{\sqrt{m}}{k} \cdot W\right) & \text { when } k=O\left(\frac{n}{\log n}\right) \\ \Theta\left(\frac{\sqrt{n}}{\sqrt{\log n}} \cdot \frac{1}{\sqrt{k}} \cdot W\right) & \text { and } k=O(m), \\ \Theta\left(\frac{r \cdot n}{a} \frac{\sqrt{m}}{k} \cdot W\right) & \text { when } k=O\left(\frac{n}{\log n}\right) \\ \Theta(W) & \text { when } k=\Omega(m), \\ & \text { and } k=O\left(\frac{n}{\log n}\right) \\ & \text { when } k=\Omega\left(\frac{n}{\log n}\right)\end{cases}
$$

Consequently, the per-flow multicast capacity (with $k-1$ receivers per source node) is the aggregated multicast capacity divided by $n$.

When the transmission range $r$ is arbitrary and the side length of the deployment region is $a$, which is no more than the critical side length for getting a connected wireless network w.h.p., we have the following results: When base stations are regularly distributed, the aggregated multicast capacity of $n$ multicast sessions is

$$
\Lambda_{k}(n)= \begin{cases}O\left(\frac{a}{r} \cdot \frac{\sqrt{m}}{k} \cdot W\right) & \text { when } k=O\left(\frac{a^{2}}{r^{2}}\right) \\ \Theta\left(\frac{a}{r} \cdot \frac{1}{\sqrt{k}} \cdot W\right) & \text { and } k=O(m), \\ \Theta\left(\frac{r \cdot n}{a} \frac{\sqrt{m}}{k} \cdot W\right) & \text { when } k=O\left(\frac{a^{2}}{r^{2}}\right) \\ & \text { when } k=\Omega(m), \\ \Theta(W) & \text { and } k=O\left(\sqrt{r^{2}}\right), \\ \text { when } k=\Omega\left(\frac{a^{2}}{r^{2}}\right)\end{cases}
$$

${ }^{1}$ We give the sufficient conditions for having a connected hybrid wireless network in section 3 .
The rest of the paper is organized as follows. In Section 2 we discuss in detail the network model used. We present an upper bound on the side length $a$ (the critical side length which leads to a connected hybrid wireless network w.h.p.) in Section 3. In Section 4 and 5 we present upper-bounds and lower-bounds respectively on multicast capacity for the hybrid wireless networks. We review the related results on network capacities in Section 6 and conclude the paper in Section 7.

\section{NETWORK MODEL}

We assume that there is a set $V=\left\{v_{1}, v_{2}, \cdots, v_{n}\right\}$ of $n$ ordinary wireless terminals randomly deployed in a square region $\Omega$, with side length $a$. Each wireless node has a transmission range $r$ such that nodes $v_{i}$ and $v_{j}$ can communicate successfully iff the Euclidean distance $\left|v_{i}-v_{j}\right| \leq r$. We further assume that there are $m$ base stations (with wireless transmission range $r$ ) $Z=\left\{z_{1}, z_{2}, \cdots, z_{m}\right\}$ regularly placed in the region $\Omega$. For example, the base stations are placed regularly at positions $\left(\frac{a}{2 \sqrt{m}}+i \frac{a}{\sqrt{m}}, \frac{a}{2 \sqrt{m}}+j \frac{a}{\sqrt{m}}\right)$ with $0 \leq i \leq \sqrt{m}-1$, and $0 \leq j \leq \sqrt{m}-1$.

Clearly, these $m$ regularly distributed base stations divide the original square region into $m$ subregions as Voronoi diagrams with side length $\frac{a}{\sqrt{m}}$. Here we generally assume that $m$ is a square of some integer for simplicity, and use $S_{i}$ to denote the subregion centered at the base station $z_{i}$. We further assume that $m=O\left(\frac{a^{2}}{r^{2}}\right)$ throughout the whole paper because of the following observation that when the number of base stations $m$ is no less than $\frac{a^{2}}{r^{2}}$, basically, the union of transmission range of $m$ base stations will cover all wireless nodes, thus a hybrid wireless network will act as a cellular network.

The complete communication graph is a graph $G=(V \cup Z, E)$, where $V=\left\{v_{1}, v_{2}, \cdots, v_{n}\right\}$ and $Z=\left\{z_{1}, z_{2}, \cdots, z_{m}\right\}$ and $E$ is the set of all possible communication links $u v$ where both $u$ and $v$ belong to $V$ or at most one of $u$ and $v$ belongs to $Z$. In this paper, we mainly assume that the transmission range $r$ is fixed and thus normalized to one unit throughout the paper. Under this assumption, the deployment region $\Omega$ will be a function of $n$.

In wireless networks, to schedule two links at the same time slot, we must ensure that the schedule avoids interference. In this paper, we mainly focus on the protocol interference model introduced in [6]. We assume that each node $v_{i}$ has a fixed interference range $R=\Theta(r)$. Under the protocol interference model, any node $v_{j}$ will be interfered by the signal from $v_{k}$ if $\left\|v_{k}-v_{j}\right\| \leq R$ where node $v_{k}$ is sending signal to some node other than $v_{j}$.

Capacity Definition: We give the formal definition of capacity in our model (also used in [10]).

Definition 1 (Feasible Rate Vector). A multicast rate vector $\lambda=\left(\lambda_{1}, \lambda_{2}, \cdots, \lambda_{n-1}, \lambda_{n}\right)$ bits/sec is feasible if there is a spatial and temporal scheme for scheduling transmissions such that by operating the network in a multi-hop fashion and buffering at intermediate nodes when awaiting transmission, every node $v_{i}$ can send $\lambda_{i}$ bits/sec average to its chosen $k-1$ destination nodes. That is, there is a $T<\infty$ such that in every time interval (with unit seconds) $[(i-1) \cdot T, i \cdot T]$, every node can send $T \cdot \lambda_{i}$ bits to its corresponding $k-1$ receivers.

DEFINITION 2 (ThroughPUT CAPACITY). An aggregated multicast throughput $\Lambda_{k}(n)$ bits/sec is feasible for multicast (each session with $k$ nodes) if there is a rate vector $\lambda=\left(\lambda_{1}, \lambda_{2}, \cdots, \lambda_{n-1}, \lambda_{n}\right)$ that is feasible and $\Lambda_{k}(n)=\sum_{i=1}^{n} \lambda_{i}$. Similarly, we say $\lambda_{k}(n)=$ $\frac{\sum_{i=1}^{n} \lambda_{i}}{n_{s}}$ is a feasible per-flow multicast throughput capacity with $n_{s}$ multicast sessions. 
DEFINITION 3 (CAPACITY OF RANDOM NETWORKS). We say that the multicast capacity per flow of a class of random networks is of order $\Theta(f(n))$ bits/sec if there are deterministic constants $c>0$ and $c<c^{\prime}<+\infty$ such that

$$
\begin{aligned}
\lim _{n \rightarrow \infty} \boldsymbol{P r}\left(\lambda_{k}(n)=c f(n) \text { is feasible }\right) & =1 \\
\liminf _{n \rightarrow \infty} \boldsymbol{P r}\left(\lambda_{k}(n)=c^{\prime} f(n) \text { is feasible }\right) & <1
\end{aligned}
$$

The aggregated multicast capacity of a class of random networks is defined similarly.

Notations: Throughout this paper, for a continuous region $\Omega$, we use $|\Omega|$ to denote its area; for a discrete set $S$, we use $|S|$ to denote its cardinality; for a tree $T$, we use $|T|$ to denote its total Euclidean edge length; $x \rightarrow \infty$ denotes that variable $x$ takes value to infinity. In addition, for simplicity we use $N_{n, m, a}$ to denote a hybrid wireless network in which there are $n$ wireless nodes randomly and uniformly distributed in the 2-dimensional unit square $\Omega$ with side length $a$, and $m$ base stations are regularly distributed in $\Omega$.

\section{THE UPPER-BOUND ON THE SIDE LENGTH $A$}

We first study the asymptotic upper-bound on the side length $a$ for both routing strategies such that the resultant network $G=$ $(V \cup Z, E)$ is connected with probability going to 1 as $n$ goes to infinity.

According to the pure ad hoc routing strategy, we know that if there are $n$ nodes, each with transmission range $r$, randomly and uniformly deployed in a square region of side length $a$, the resulting network will be connected with probability at least $e^{e^{-\beta}}$ when $\frac{a}{r} \leq$ $\sqrt{\frac{n \pi}{\log n+\beta}}$ for $\beta \rightarrow \infty$ from the result of [10].

Next, we show the upper bound of the side length $a$ when pure hybrid routing is used. Obviously, when the base stations are used in the communications, the whole network is connected iff all nodes (including the base station) falling in each subregion form a connected subnetwork. Let $n_{i}$ denote the number of nodes falling in subregion $S_{i}$ (including $z_{i}$ ) and $M_{i}$ be the length of longest edge of the Euclidean minimum spanning tree of nodes in $S_{i}$. For a set of nodes, the critical transmitting range (CTR) for connectivity is always the length of the longest edge of the Euclidean minimum spanning tree (EMST) of this set of nodes. Based on the assumption that $m=o\left(\frac{a^{2}}{r^{2}}\right)$, we show the following lemma first:

LEMMA 1. There is a sequence of $\delta(n) \rightarrow 0$ such that $\boldsymbol{P r}\left(\forall i: n_{i} \geq \Theta\left(\frac{n}{m}\right)\right)>1-\delta(n)$ when $m=O\left(\frac{n}{\log n}\right)$. Here $1 \leq i \leq m$.

ProOF. Let $C$ be the class of axis-aligned squares of side-length $\frac{a}{\sqrt{m}}$. Notice that the probability that a node falls in such a square is $\frac{1}{m}$. In addition, we know the VC-dimension of $C$ is 3 from the result in [10]. Hence, for any subregion $S$, by VC-Theorem,

$$
\begin{gathered}
\operatorname{Pr}\left(\sup _{S \in C}\left|\frac{\# \text { of nodes in } S}{n}-\frac{1}{m}\right| \leq \epsilon(n)\right)>1-\delta(n) \\
\text { whenever } n \geq \max \left\{\frac{32}{\epsilon(n)} \cdot \log \frac{13}{\epsilon(n)}, \frac{4}{\epsilon(n)} \log \frac{2}{\delta(n)}\right\}
\end{gathered}
$$

This condition is satisfied when $\epsilon(n)=\frac{1}{2 m}, \delta(n)=\frac{2}{n}$. Thus

$$
\operatorname{Pr}\left(\sup _{S \in C}\left\{\# \text { of nodes in } S \geq \frac{n}{2 m}\right\}\right)>1-\frac{2}{n} \text {. }
$$

Hence, $\operatorname{Pr}\left(\forall i: n_{i} \geq \Theta\left(\frac{n}{m}\right)\right)>1-\frac{2}{n}$.

THEOREM 2. Assume in hybrid wireless network $N_{n, m, a}$, each node has transmission range $r$. When $\frac{a}{r} \leq \sqrt{\frac{c n \pi}{\log \left(c \frac{n}{m}\right)+\beta}}$ for $\beta \rightarrow \infty$, the resulting network $G=(V \cup Z, E)$ is connected with probability at least $\frac{1}{e^{e^{-\beta}}}$, which goes to 1 if $\beta \rightarrow \infty$.

PROOF. According to the result of $[10,12]$, we know for any $\beta$,

$$
\lim _{n_{i} \rightarrow \infty} \operatorname{Pr}\left(n_{i} \pi \cdot\left(\frac{M_{n_{i}, a, m}}{\frac{a}{\sqrt{m}}}\right)^{2}-\log n_{i} \leq \beta\right)=\frac{1}{e^{e^{-\beta}}} .
$$

Let $n_{i}$ be the number of points distributed uniformly at random in the 2-dimensional square with side length $\frac{a}{\sqrt{m}}$, and $M_{n_{i}, a, m}$ be the random variable denoting the length of the longest edge of EMST built on this set of $n_{i}$ nodes. Assume $n_{i}=c \cdot \frac{n}{m}$ for some constant $c$ from Lemma 1, then with probability $\frac{1}{e^{e^{-\beta}}}$, the longest edge length $M_{n_{i}, a, m}$ is at most $\sqrt{\frac{\beta+\log \left(c \frac{n}{m}\right)}{c n \pi}} \cdot a$. Thus, when $\beta \rightarrow$ $\infty$ and $a \leq \sqrt{\frac{c n \pi}{\log \left(\frac{n}{m}\right)+\beta}} \cdot r$, we know that the longest edge of EMST has length at most $r$ almost surely. For example, we can set $a=r \sqrt{\frac{c n}{\log n}}$ where $\beta=(\pi-1) \log n+\log \frac{m}{c}$.

\section{UPPER BOUNDS ON MULTICAST CA- PACITY}

\subsection{When $k=O(m)$ and $k \geq \sqrt{8 m \ln n}$}

In this section, we first study the multicast capacity of the hybrid wireless network when the number of receivers $k=O(m)$ which is at most $O\left(a^{2} / r^{2}\right)$. For each source node $v_{i}$, when we multicast the data from $v_{i}$ to all its $k-1$ receivers in node set $U_{i}=\left\{v_{i_{1}}, v_{i_{2}}, \cdots, v_{i_{k-1}}\right\}$, the resulting multicast tree will contain at least $k$ nodes. More possibly, when a non-leaf node $v_{j}$ in the multicast tree sends data to its children, all nodes within its transmission range will receive the data or at least they cannot transmit simultaneously. In this case, we say all these nodes are charged a copy of the data. Generally, let $C$ be the number of nodes that will get a copy of the data when $k-1$ receivers are randomly selected for each possible source node $v$, the total multicast capacity is at most, w.h.p., $\frac{n \cdot W}{C}$.

The rest of the subsection is devoted to give a better lower bound on $C$, thus get a tighter upper bound of multicast capacity of the hybrid wireless network. For simplicity, hereafter when we say $k$ receivers, we mean that one source node pluses all its $k-1$ receivers.

Assume $k$ receivers are randomly distributed in the square region with side length $a$, which is regularly divided by $m$ base stations, we show that the length of resulting multicast tree (or say forest) has some lower bound.

LEMMA 3. Assume $s$ is the number of subregions which contain at least one of $k$ receivers, then $s=\Theta(k)$ with high probability when $k \geq \sqrt{8 m \ln n}$.

PROOF. Let $y_{j}=\{0,1\}$ be an indicator denoting whether subregion $S_{j}$ contains at least one receiver. Clearly, $s=\sum_{j=1}^{j=m} y_{j}$ and the expected value $E(s)$ of $s$ is

$$
\begin{aligned}
& E(s)=m\left(1-\left(1-\frac{1}{m}\right)^{k}\right) \geq m\left(1-e^{-\frac{k}{m}}\right) \geq m \frac{k}{2 m}=\frac{k}{2} \\
& \text { because } \operatorname{Pr}\left(y_{j}=1\right)=1-\left(1-\frac{1}{m}\right)^{k} .
\end{aligned}
$$


Consequently, according to Binomial Distribution, we have

$\operatorname{Pr}\left(s \leq \frac{k}{4}\right) \leq e^{\frac{-2(E(s)-b)^{2}}{m}} \leq e^{-2\left(\frac{k}{2}-\frac{k}{4}\right)^{2}}=e^{-\frac{k^{2}}{8 m}} \rightarrow 0$

So, $s \geq \frac{k}{4}$ with a probability $\geq 1-e^{-\frac{k^{2}}{8 m}} \geq 1-\frac{1}{n}$, and we also know $s$ is at most $k$. Thus $s=\Theta(k)$ with probability at least $1-\frac{1}{n}$. We assume $s=c_{0} k$ for some constant $c_{0}$.

LEMMA 4. Assume $S_{i}$ is a subregion containing at least one of $k$ receivers, then the expected length $L_{i}$ between the base station and one receiver is $c_{1} \cdot \frac{a}{\sqrt{m}}$, here $\frac{\pi}{12}<c_{1}<\frac{\sqrt{2} \pi}{6}$.

PROOF. Assume the node is $u$ and the base station is $z_{i}$. Thus the expected length $E\left(L_{i}\right)=E\left(\left\|u z_{i}\right\|\right)$. Then, $E\left(\left\|u z_{i}\right\|\right)>$ $\int_{0}^{\frac{a}{2 \sqrt{m}}} \frac{2 \pi r}{\left(\frac{a}{\sqrt{m}}\right)^{2}} \cdot r d r$. So $E\left(\left\|u z_{i}\right\|\right)>\frac{\pi}{12} \cdot \frac{a}{\sqrt{m}}$. On the other hand, we have $E\left(\left\|u z_{i}\right\|\right)<\int_{0}^{\frac{a}{\sqrt{2 m}}} \frac{2 \pi r}{\left(\frac{a}{\sqrt{m}}\right)^{2}} \cdot r d r$. So $E\left(\left\|u z_{i}\right\|\right)<$ $\frac{\sqrt{2} \pi}{6} \cdot \frac{a}{\sqrt{m}}$. This finishes the proof.

Next, we show the bound of total length of the multicast tree (forest) $T$ spanning all $k-1$ receivers for source node $v$.

LEMMA 5. In hybrid wireless network $N_{n, m, a}$, for the source node $v$, we randomly pick $k-1$ nodes from remaining $n-1$ nodes as receivers of $v$. Then, the total length $L$ of the multicast tree $T$ spanning these $k$ nodes is at least $\frac{c_{1} \cdot c_{0}}{2}\left(\frac{a}{\sqrt{m}}\right) \cdot k$ and at most $\frac{3 c_{1} \cdot c_{0}}{2}\left(\frac{a}{\sqrt{m}}\right) \cdot k$ w.h.p.

PROOF. Let $L_{i}$ denote the length of subtree for connecting all receivers in subregion $S_{i}$. Then the total length $L$ of multicast tree consisting of these subtrees satisfies $E(L)=E\left(\sum_{i=1}^{i=m} L_{i}\right) \geq$ $c_{1} \frac{a}{\sqrt{m}} \cdot s$ from Lemma 4. Here, $s$ is the number of subregions which contain at least one receiver. Clearly, for some constant $c_{2}$,

$$
\operatorname{Var}(L)=\operatorname{Var}\left(\sum_{i=1}^{i=m} L_{i}\right) \leq c_{2}\left(\frac{a}{\sqrt{m}}\right)^{2} s
$$

From $\operatorname{Pr}(L-E(L) \geq A) \leq \frac{\operatorname{Var}(L)}{A^{2}}$, we have

$\operatorname{Pr}\left(\left|L-c_{1} \frac{a}{\sqrt{m}} s\right|>\frac{c_{1}}{2} \frac{a}{\sqrt{m}} s\right) \leq \frac{c_{2}\left(\frac{a}{\sqrt{m}}\right)^{2} s}{\frac{c_{1}^{2}}{4}\left(\frac{a}{\sqrt{m}}\right)^{2} s^{2}}=\frac{4 c_{2}}{c_{1}^{2}} \frac{1}{s} \rightarrow 0$.

Thus, we have

$\boldsymbol{P r}\left(\frac{c_{1} c_{0}}{2}\left(\frac{a}{\sqrt{m}}\right) k \leq L \leq \frac{3 c_{1} c_{0}}{2}\left(\frac{a}{\sqrt{m}}\right) k\right)=1-\frac{4 c_{2}}{c_{1}^{2} \cdot c_{0}} \frac{1}{k} \rightarrow 1$

when $k$ goes to $\infty$. Here $s=c_{0} k$ for some constant $c_{0}$ from Lemma 3.

For each source node $v$, assume $T_{i}$ is the tree spanning all receivers falling into subregion $S_{i}$, then multicast tree (forest) $T$ is the union of all such sub-multicast trees, i.e., $T=\cup_{i=q}^{m} T_{i}$. Given the length $|T|$ of multicast tree (forest) $T$ spanning $k$ nodes, we know the lower-bound on the number of nodes (including leaf nodes) $C$ in $T$ is at least $\frac{|T|}{r}$.

Actually we know that when nodes on the multicast tree relay data from the source node to all receivers, not only their downstream nodes that will get a copy of data, but all their neighboring nodes will get one copy of the data. We then give a tighter bound for $C$ by analyzing the number of nodes which will get a copy of data.

Given a multicast tree (forest) $T$, let $|D(T)|$ denote the region covered by all transmitting disks of all transmitting nodes (internal nodes of T), we first prove that $|D(T)|$ is at least $\frac{c_{2} \cdot k \cdot \frac{a}{\sqrt{m}} \cdot r}{c_{0}}$ w.h.p.
Lemma 6. The area of the region $D(T)$, denoted by $|D(T)|$, w.h.p., is at least $\frac{c_{2} \cdot k \cdot \frac{a}{\sqrt{m}} \cdot r}{c_{0}}$ when the number of receivers $k<$ $\theta_{1} \cdot \frac{a^{2}}{r^{2}}$ for some constant $\theta_{1}$.

PROOF. For any multicast tree $T$ spanning all $k$ receivers, we can use the similar methods as in [10] to build a connected dominate set (CDS), and make sure the source node and all base stations needed will be selected into the CDS, and construct a multicast tree $T^{\prime}$ which is rooted at the source node $s$ by a simple breadth-firstsearch computed from the CDS.

Based on Lemma 6, we show the following lemma:

LEMMA 7. With high probability, the number $C$ of nodes that get a copy of the multicast data is $>\frac{c_{2} \cdot k \cdot r \cdot n}{2 c_{0} \sqrt{m a}}$ when $\frac{k}{\sqrt{m}} \rightarrow \infty$.

Proof. Consider a multicast tree $T$. Let $X_{i}=\{0,1\}$ be an indicator variable denoting whether the $i$ th node $v_{i}$ falls inside the region $D(T)$ for a multicast tree $T$. Thus, $\operatorname{Pr}\left(X_{i}=1\right)=\frac{|D(T)|}{a^{2}}$. Recall that, we already proved that $|D(T)| \geq \frac{c_{2} \cdot k \cdot \frac{a}{\sqrt{m}} \cdot r}{c_{0}}$ w.h.p.. Thus, we have

$$
\operatorname{Pr}\left(X_{i}=1\right) \geq \frac{c_{2} \cdot k \cdot r}{c_{0} \sqrt{m} a}
$$

Clearly, $X=\sum_{i=1}^{n} X_{i}$ is the expected number of nodes falling inside the region $D(T)$, which is also the number $C$ of nodes that will get a copy of the data by multicast. Then $E(X) \geq \frac{c_{2} \cdot k \cdot r \cdot n}{c_{0} \sqrt{m} a}$.

From the Hoeffding's inequality, $\operatorname{Pr}(X \leq b) \leq e^{\frac{-2(n \cdot p-b)^{2}}{n}}$, where constant $0<b \leq n \cdot p$. By letting $b=n \cdot \frac{|\bar{D}(T)|}{2 a^{2}}$, we have

$\operatorname{Pr}\left(C \leq n \cdot \frac{|D(T)|}{2 a^{2}}\right) \leq e^{-\frac{n \cdot\left(c_{2}\right)^{2} \cdot k^{2} \cdot r^{2}}{2\left(c_{0}\right)^{2} \cdot m \cdot a^{2}}} \leq e^{-\frac{\left(c_{2}\right)^{2} \cdot k^{2} \cdot \log n}{2 \pi c_{0}^{2} \cdot m}}$

The last formula is $=\frac{1}{n^{\frac{\left(c_{2}\right)^{2} \cdot k^{2}}{2 \pi c_{0}^{2} \cdot m}}}$, will go to 0 when $n$ goes to $\infty$ and $\frac{k}{\sqrt{m}} \rightarrow \infty$. Thus, from Lemma 6 , when $n \rightarrow \infty$ and $\frac{k}{\sqrt{m}} \rightarrow \infty$, we have $\operatorname{Pr}\left(C>\frac{c_{2} \cdot r \cdot k \cdot n}{2 c_{0} \cdot \sqrt{m} \cdot a}\right) \geq \operatorname{Pr}\left(C>n \cdot \frac{D(T)}{2 a^{2}}\right) \rightarrow 1$.

THEOREM 8. The multicast capacity with $k-1$ receivers for each source node of n nodes in hybrid wireless network $N_{n, m, a}$, is at most $c_{3} \cdot \frac{a}{r} \cdot \frac{W}{k} \cdot \sqrt{m}$ for some constant $c_{3}$ when $k=O(m)$ and $k=\sqrt{\Omega(m \ln n)}$.

PROOF. Notice that the multicast capacity is at most $\frac{n W}{C}$ and $C \geq \frac{c_{2} \cdot r \cdot k \cdot n}{2 c_{0} \cdot \sqrt{m} \cdot a}$ w.h.p. when $k<\theta_{1} \cdot a^{2} / r^{2}$. Thus, the multicast capacity $\Lambda_{k}(n)$ is at most

$$
\frac{n W \cdot 2 c_{0} \sqrt{m} a}{c_{2} \cdot r \cdot k \cdot n}=c_{3} \cdot \frac{a}{r} \cdot \frac{W}{k} \cdot \sqrt{m}
$$

for a constant $c_{3}=\frac{2 c_{0}}{c_{2}}$. This finishes the proof.

To guarantee that we have a connected network w.h.p., we need $a \leq r \sqrt{\frac{n \pi}{\log \left(c \cdot \frac{n}{m}\right)+\beta}}$ for $\beta \rightarrow \infty$. Thus, if $m=o(\sqrt{n})$ and we let $\gamma=c_{3} \sqrt{\pi}$, we have the following corollary:

COROLLARY 9. The multicast capacity for a random hybrid wireless network $N_{n, m, a}$, when $k=O(m)$ and $k=\sqrt{\Omega(m \ln n)}$, is at most

$$
\Lambda_{k}(n) \leq \gamma \cdot \frac{\sqrt{n}}{\sqrt{\log n} \cdot \sqrt{k}} \cdot W=O\left(\frac{\sqrt{n}}{\sqrt{\log n}} \cdot \frac{\sqrt{m}}{k} \cdot W\right) .
$$

Consequently, the multicast capacity per flow is

$$
\lambda_{k}(n)=\frac{\Lambda_{k}(n)}{n}=O\left(\frac{1}{\sqrt{n \log n}} \cdot \frac{\sqrt{m}}{k} \cdot W\right) .
$$




\subsection{When $k=O\left(a^{2} / r^{2}\right)$ and $\frac{m}{k} \rightarrow 0$}

In this subsection, we give an upper bound on the multicast capacity when the number of base stations is much smaller than the number of receivers.

LEMMA 10. When the number of receivers $k=O\left(\frac{a^{2}}{r^{2}}\right)$ and $\frac{k}{m} \rightarrow \infty$, the number of subregions which contain at least one receiver is bigger than $c_{4} \cdot m$ with probability at least $1-e^{-\left(2 c_{4}\right)^{2} \cdot m}$ which goes to 1 when $m \rightarrow \infty$, here $c_{4}$ is a constant which is less than 1 .

PROOF. The proof procedure is similar as that of Lemma 3.

In addition, we assume that the subregion $S_{i}$ contains $k_{i}$ receivers. Clearly, we have $k=\sum_{i=1}^{i=m} k_{i}$. According to the result in [10], we know that the expected length of spanning tree $L_{i}$ covering $k_{i}$ potential receivers in a subregion with length $\frac{a}{\sqrt{m}}$ satisfies $L_{i} \geq \tau \frac{a}{\sqrt{m}} \sqrt{k}_{i}$ when $k_{i} \rightarrow \infty$. Here $\tau$ is a constant. Next, we prove that the Euclidean length of multicast tree $T$ has a bigger lower bound.

LEMMA 11. The total length $L$ of the multicast tree (forest) $T$ which spans all $k$ receivers among all $m$ subregions is at least $\delta_{2}$. $a \cdot \sqrt{k}$, when $k=O\left(a^{2} / r^{2}\right), k=\Omega(m)$ and $\frac{m}{k} \rightarrow 0$, here $\delta_{2}$ is a constant.

PROOF. For any subregion $S_{i}$ which contains at least one receiver, we let $q_{i j} \in\{0,1\}$ be an indicator variable denoting whether the $j^{t h}$ receiver will fall inside $S_{i}$, here $1 \leq i \leq m$ and $1 \leq j \leq k$. Hence, $k_{i}=\sum_{j=1}^{j=k} q_{i j}$ is the number of receivers falling inside the subregion $S_{i}$.

Clearly, $\operatorname{Var}\left(q_{i j}\right)=\frac{1}{m}\left(1-\frac{1}{m}\right)$, then

$$
\operatorname{Var}\left(k_{i}\right)=\sum_{j=1}^{j=k} \operatorname{Var}\left(q_{i j}\right)=\frac{k}{m}\left(1-\frac{1}{m}\right)
$$

From Chebyshev's Inequality, we have

$$
\operatorname{Pr}\left(\left|k_{i}-E\left(k_{i}\right)\right|>\delta E\left(k_{i}\right)\right) \leq \frac{\operatorname{Var}\left(k_{i}\right)}{\delta^{2} E\left(k_{i}\right)^{2}}=\delta \frac{m-1}{k} \rightarrow 0
$$

because $\frac{m}{k} \rightarrow 0$ and $E\left(k_{i}\right)=\frac{k}{m}$. Here $\delta$ is a constant. Thus,

$$
(1-\delta) \frac{k}{m}<k_{i}<(1+\delta) \frac{k}{m}
$$

with probability at least $1-\delta \cdot \frac{m-1}{k}$, which goes to 1 when $\frac{k}{m} \rightarrow \infty$. Thus, the length of multicast tree $T, L=\sum_{i=1}^{m} L_{i}$ satisfies

$$
L \geq \tau \frac{a}{\sqrt{m}} \sqrt{(1-\delta) \frac{k}{m}} \times c_{4} m
$$

with probability $\left(1-\delta \frac{m-1}{k}\right) \cdot\left(1-e^{-\left(2 c_{4}\right)^{2} \cdot m}\right)$, which goes to 1 when $\frac{k}{m} \rightarrow \infty$ and $m \rightarrow \infty$. By letting $\delta_{2}=c_{4} \tau \sqrt{1-\delta}$, we finish the proof.

LEMMA 12. The total area covered by tree $T$ is at least $c_{7} \cdot \sqrt{k}$. $a \cdot r$, here $c_{7}$ is a constant, when $k=O\left(a^{2} / r^{2}\right)$ and $k=\Omega(m)$, $\frac{m}{k} \rightarrow 0$.

Proof. Assume $\left|D\left(T_{i}\right)\right|$ denotes the area covered by all transmitting disks of all transmitting nodes (internal nodes) in the multicast tree $T_{i}$. As shown before, $L_{i} \geq \tau \frac{a}{\sqrt{m}} \sqrt{k}_{i}$ and $(1-\delta) \frac{k}{m}<k_{i}$, then based on the proof of Lemma 6, we get

$$
\left|D\left(T_{i}\right)\right|>\frac{\tau \sqrt{(1-\delta) \frac{k}{m}} \frac{a}{\sqrt{m}} r}{c_{0}}
$$

Consider one subregion $S_{i}$, it has at most 8 neighbor subregions. So the area covered by the tree $T_{i}$ in the subregion $S_{i}$ overlapping with other subregions (possibly 4 rectangles with area $r \cdot \frac{a}{\sqrt{m}}$ and 4 sectors with radius $r$ ), is at most $4 \frac{a}{\sqrt{m}} \cdot r+4 \pi \cdot r^{2}$. From Lemma 3 , the total area of the multicast tree (forest) $T$ satisfies:

$$
\begin{aligned}
|D(T)| & \geq c_{4} \cdot m \cdot\left|D\left(T_{i}\right)\right|-\left(4 \frac{a}{\sqrt{m}} \cdot r+4 \pi \cdot r^{2}\right) \cdot m \\
& \geq \frac{c_{4} \cdot \tau \cdot \sqrt{1-\delta} \cdot \sqrt{k} \cdot a \cdot r}{c_{0}}
\end{aligned}
$$

We finish the proof by letting $c_{7}=\frac{c_{4} \cdot \tau \cdot \sqrt{1-\delta}}{c_{0}}$.

Consequently we have the following lemma.

LEMMA 13. The number $C$ of nodes that get a copy of the multicast data satisfies $C \geq \frac{c_{7} \cdot \sqrt{k} \cdot r \cdot n}{2 a}$ w.h.p. when $n$ and $k$ go to $\infty$

Proof. Let $X_{j}=\{0,1\}$ be an indicator variable denoting whether the $j$ th node $v_{j}$ will fall inside the region $D(T)$ for a multicast tree $T$. Clearly, we have $\operatorname{Pr}\left(X_{j}=1\right)=\frac{|D(T)|}{a^{2}} \geq$ $\frac{c_{7} \cdot \sqrt{k} \cdot r}{a}$ from Lemma 12. Thus, the expected number of node $X=$ $\sum_{j=1}^{n} X_{j}$ falling into $|D(T)|$ is

$$
E(X) \geq \frac{c_{7} \cdot \sqrt{k} \cdot r \cdot n}{a}
$$

According to the Hoeffding's inequality and by letting $b=n$. $\frac{|D(T)|}{2 a^{2}}$, we have

$$
\begin{gathered}
\operatorname{Pr}\left(C \leq n \cdot \frac{|D(T)|}{2 a^{2}}\right) \leq e^{\frac{-2\left(n \cdot \frac{|D(T)|}{a^{2}}-n \cdot \frac{|D(T)|}{2 a^{2}}\right)^{2}}{n}} \\
\leq e^{-\frac{c_{7}^{2} \cdot k \cdot n \cdot r^{2}}{2 a^{2}}} \leq e^{-\frac{c_{7}^{2} \cdot k \cdot \log n}{2 a^{2}}}=\frac{1}{n^{\frac{c_{7}^{2} \cdot k}{2}}} \rightarrow 0
\end{gathered}
$$

when both $n$ and $k$ go to $\infty$. The last inequality comes from that $a \leq r \sqrt{\frac{n \pi}{\log c \cdot \frac{n}{m}+\beta}}$. Thus, $\operatorname{Pr}\left(C \geq \frac{c_{7} \cdot \sqrt{k} \cdot r \cdot n}{2 a}\right) \rightarrow 1$ when $n$ and $k$ go to $\infty$. This finishes the proof.

Finally, through Theorem 8 and Corollary 9, we have the following results:

THEOREM 14. The multicast capacity with $k-1$ receivers for each source node of n nodes in hybrid wireless network $N_{n, m, a}$, is at most $\frac{2 a W}{c_{7} \cdot r \sqrt{k}}$ for some constant $c_{7}$ when $k=O\left(a^{2} / r^{2}\right)$ and $\frac{m}{k}$ $\rightarrow 0$.

The theorem follows the following observation. Clearly, $\Lambda_{k}(n)$ is at most

$$
\frac{n \cdot W}{C} \leq \frac{2 n W \cdot a}{c_{7} \cdot r \sqrt{k} \cdot n}=\frac{2 a W}{c_{7} \cdot r \sqrt{k}}
$$

Or we use the other formula to the multicast capacity as

$$
\Lambda_{k}(n)=O\left(\frac{\sqrt{n}}{\sqrt{\log n} \cdot \sqrt{k}} \cdot W\right)
$$

Consequently, the multicast capacity per flow is

$$
\lambda_{k}(n)=\frac{\Lambda_{k}(n)}{n}=O\left(\frac{1}{\sqrt{n \log n} \cdot \sqrt{k}} \cdot W\right)
$$




\subsection{When $k=o(\sqrt{m})$}

Clearly, when $k=o(\sqrt{m})$, the expected number of subregions which contain at least one receiver, $E(s)=k w . h . p$. from Lemma 3.

LEMMA 15 . When $k=o(\sqrt{m})$ and $m \rightarrow \infty$, for any subregion $S_{i}$ which contains at least one receiver, $S_{i}$ contains exactly one receiver with high probability.

PROOF. $\forall S_{i}$, assume $k_{i}$ is the number of receivers in $S_{i}$, then the probability for $S_{i}$ containing at least one receiver is

$$
\operatorname{Pr}\left(k_{i} \geq 1\right)=1-\left(1-\frac{1}{m}\right)^{k}
$$

In addition, the probability for $S_{i}$ containing at least two receivers is

$$
\operatorname{Pr}\left(k_{i} \geq 2\right)=1-\left(1-\frac{1}{m}\right)^{k}-k \cdot\left(1-\frac{1}{m}\right)^{k-1} \cdot \frac{1}{m}
$$

Then for any subregion $S_{i}$ which contains at least one receiver, the probability for $S_{i}$ containing at least two receivers is

$$
\operatorname{Pr}\left(k_{i} \geq 2 \mid k_{i} \geq 1\right)=1-\frac{\frac{k}{m}\left(1-\frac{1}{m}\right)^{k-1}}{1-\left(1-\frac{1}{m}\right)^{k}}
$$

In addition, we know when $m \rightarrow \infty$ and $\frac{k}{m} \rightarrow 0$ from $k=$ $o(\sqrt{m})$. Assume $x=\frac{k}{m}$, so we have

$$
\begin{aligned}
\lim _{\frac{k}{m} \rightarrow 0 \text { and } m \rightarrow \infty} \frac{\frac{k}{m}\left(1-\frac{1}{m}\right)^{k-1}}{1-\left(1-\frac{1}{m}\right)^{k}} & =\lim _{x \rightarrow 0 \text { and } m \rightarrow \infty} \frac{x \cdot \frac{1}{1-\frac{1}{m}} \cdot e^{-x}}{1-e^{-x}} \\
& =\lim _{x \rightarrow 0} 1-x=1
\end{aligned}
$$

So, when $\frac{k}{m} \rightarrow 0$ and $m \rightarrow \infty, \operatorname{Pr}\left(k_{i} \geq 2 \mid k_{i} \geq 1\right)$ goes to 0 . This finishes proof.

We then prove the lower bound of the length of multicast tree (forest) $T$ of source node $v$ by the following lemma.

LEMMA 16. For any source node $v$, the length of multicast tree (forest) $T$ which spans all $k$ receivers of $v$ is at least $\frac{c_{3} \cdot a \cdot k}{2 \sqrt{m}}$ with probability at least $1-\frac{\pi}{2 \cdot k}$, which goes to 1 when $k \rightarrow \infty$ and $k=O\left(\frac{a^{2}}{r^{2}}\right)$ and $k=o(\sqrt{m})$.

PROOF. For any source node $v$, we know that there are $k$ subregions containing one of $k$ receivers w.h.p. from Lemma 15. Assume $\bar{L}=\frac{\sum_{i=1}^{k} L_{i}}{k}$ which denotes the mean of the total Euclidean length for all $k$ pairs of base stations/receivers. Then according to the Weak Law of Large numbers,

$$
\operatorname{Pr}(|\bar{L}-\mu|<\epsilon) \geq 1-\frac{\delta^{2}}{k \cdot \epsilon^{2}}
$$

By letting $\mu$ be the expected value of $E\left(L_{i}\right)\left(E\left(L_{i}\right)=c_{3} \frac{a}{\sqrt{m}}\right.$ by Lemma 4) and letting $\epsilon=\frac{1}{2} E\left(L_{i}\right)$ we have

$$
\begin{aligned}
\operatorname{Pr}\left(\left|\bar{L}-E\left(L_{i}\right)\right|<\frac{1}{2} E\left(L_{i}\right)\right) & \geq 1-\frac{\delta^{2}}{k \cdot\left(\frac{1}{2} E\left(L_{i}\right)\right)^{2}} \\
\geq 1-\frac{\frac{\pi}{8} \cdot c_{3}^{2}\left(\frac{a}{\sqrt{m}}\right)^{2}}{k \cdot \frac{1}{4} c_{3}^{2}\left(\frac{a}{\sqrt{m}}\right)^{2}} & =1-\frac{\pi}{2 \cdot k}
\end{aligned}
$$

which goes to 1 when $k \rightarrow \infty$. Here, $\delta^{2}=\operatorname{Var}\left(L_{i}\right)=E\left(L_{i}^{2}\right)-$ $E^{2}\left(L_{i}\right)$ thus is less than

$$
\int_{0}^{\frac{a}{\sqrt{2 m}}} \frac{2 \pi r}{\left(\frac{a}{\sqrt{m}}\right)^{2}} \cdot r^{2} d r-\left(c_{3} \frac{a}{\sqrt{m}}\right)^{2}=\left(\frac{\pi}{8}-c_{3}^{2}\right)\left(\frac{a}{\sqrt{m}}\right)^{2}
$$

from Lemma 4. So, we know the total Euclidean length $L=$ $\sum_{i=1}^{k} L_{i}$ satisfies

$$
\frac{c_{3} \cdot a \cdot k}{2 \sqrt{m}}<L<\frac{3 c_{3} \cdot a \cdot k}{2 \sqrt{m}}
$$

w.h.p. This finishes proof.

Hence, the total expected number of nodes $C_{j}$ (existing on the tree $T_{j}$ ) which receives one copy of data from source $v_{j}$ satisfies

$$
C_{j} \geq \frac{\left|T_{j}\right|}{r} \geq \frac{c_{3}}{2} \frac{a}{\sqrt{m} \cdot r} \cdot k
$$

w.h.p. Thus we have the following theorem;

THEOREM 17. The multicast capacity for hybrid wireless network $N_{n, m, a}$ is at most $\frac{2}{c_{3}} \frac{r \cdot n}{a} \frac{\sqrt{m}}{k} \cdot W$ when $k=o(\sqrt{m})$.

PROOF. As we know, for $n_{s}$ source nodes and each of them can transmit data by rate at most $W$ bits/sec, the total bits transmitted through the network per second cannot exceed $n_{s} \cdot W$, thus $\sum_{j=1}^{n_{s}} \lambda_{j} \cdot C_{j} \leq n_{s} \cdot W$. Hence,

$$
\sum_{j=1}^{n_{s}} \lambda_{j} \leq \frac{n_{s} \cdot W}{\frac{c_{3}}{2} \frac{a}{\sqrt{m} \cdot r} \cdot k}=\frac{2}{c_{3}} \frac{r \cdot n_{s}}{a} \frac{\sqrt{m}}{k} \cdot W
$$

when the number of source node is equal to $n$.

\subsection{When $k=\Omega\left(\frac{a^{2}}{r^{2}}\right)$}

In [10], Li has proved that when $k=\Omega\left(\frac{a^{2}}{r^{2}}\right)$, the union of the transmission disks of these $k$ receiver nodes in a multicast will cover at least a constant fraction, say $0<\rho_{2} \leq 1$, of the deployment region. Thus the capacity of hybrid wireless network will approximately be equal to the broadcast capacity. So we have the following theorem:

THEOREM 18. When $k \geq \theta \cdot a^{2} / r^{2}$ for a constant $\theta$, the total multicast capacity $\Lambda_{k}(n)$ of all nodes is bounded from above by

$$
\Lambda_{k}(n) \leq \frac{W \cdot a^{2}}{\rho_{2} a^{2}}=\frac{W}{\rho_{2}}=O(W)
$$

where $\rho_{2}$ is a constant depending only on $\theta$.

\section{LOWER BOUNDS ON MULTICAST CA- PACITY}

In this section, we will derive lower bounds on the multicast capacity $\Lambda_{k}(n)$. Specifically, we will provide a multicast scheme and prove that the multicast capacity achieved by our scheme matches the asymptotic upper bounds.

\subsection{Good Approximation of MCDS}

We use the similar idea as [10] to get a multicast scheme which is based on a good approximation of a minimum connected dominating set (MCDS) of a random network. First, we partition the region with side length $a$ into squarelets, each with side length $\frac{r}{\sqrt{5}}$, here $r$ is the transmission range. We can guarantee two things by this partition:(1) Any two nodes from two squarelets (sharing a common side) will be able to communicate with each other directly; (2) Every squarelet contains at least one node almost surely (Results in [10]). Then if we randomly pick up one node from each squarelet, clearly, these nodes will automatically form a dominating set.

Given the CDS, for any node $v$ in it, if we let $\Delta$ be the maximum number of nodes in CDS whose transmission will interfere with the 
transmission of $v$ in CDS, then by using the area argument, we can show that $\Delta \leq \frac{\pi \cdot(R+2 r)^{2}}{r^{2} / 5}=5 \pi\left(2+\frac{R}{r}\right)^{2}$.

Clearly, this property ensures that we can schedule the transmissions of all nodes in CDS by a TDMA manner such that all nodes will be able to transmit at least once in every $\Delta$ time slots, that guarantees that for any node in the CDS in some subregion, it can transmit at least once in every $\Delta$ time slots regardless of our two candidate routing strategies.

\subsection{When $k=O(m)$ and $\frac{k}{\sqrt{m}} \rightarrow \infty$}

When the number of receivers, plus the source node, $k$ is at most $\theta_{1} \frac{a^{2}}{r^{2}}$, we will construct a multicast tree in each subregion $S_{i}$ spanning $k_{i}$ receivers inside and thus obtain a multicast tree (forest) which spans all $k$ receivers.

Consider an instance of a random network $G=(V \cup Z, E)$ and also an instance of multicast with $v_{j}$ as the source node and $U_{j}=$ $\left\{v_{j_{1}}, v_{j_{2}}, v_{j_{3}}, \cdots, v_{j_{k}}\right\}$ as the receiver nodes plus the source node. Here for simplicity we assume $v_{j}=v_{j_{1}}$. Let $U_{j}^{i}=\left\{v_{j_{1}}^{i}, v_{j_{2}}^{i}, \cdots\right.$, $\left.v_{j_{t}}^{i}, z_{i}\right\}$ denote all the receivers of source node $v_{j}$ existing in the subregion $S_{i}$ plus the base station $z_{i}$. Here $t$ is the number of receivers of source node $v_{j}$ existing in the subregion $S_{i}$. We will construct a multicast structure for $U_{j}^{i}$ by Algorithm 1 .

\footnotetext{
$\overline{\text { Algorithm } 1 \text { Multicast Routing for Nodes } U_{j}^{i} \text { inside subregion } S_{i}}$

1: for each subregion $S_{i}$ do

2: We partition the deployment square into squarelets, each with side length $r / \sqrt{5}$ (as in [14]. Thus, for subregion $S_{i}$, we have $\left\lceil\frac{5 a^{2}}{m \cdot r^{2}}\right\rceil$ squarelets. Each squarelet is denoted by $\left(l^{1}, l^{2}\right)$ when it is the $l^{1}$ th column and $l^{2}$ th row.

3: Let $P_{j_{i}}=\left\{p_{j, 1}^{i}, p_{j, 2}^{i}, \cdots, p_{j, t}^{i}, p^{i}\right\}$ be the set of randomly and independently selected points used to find the terminals $\left.U_{j}^{i}=\left\{v_{j_{1}}^{i}, v_{j_{2}}^{i}, \cdots, v_{j_{t}}^{i}, z_{i}\right\}\right\}$, here $p^{i}$ denote the position of the base station $z_{i}$. Recall that for any $1 \leq t \leq\left|U_{j}^{i}\right|-1$, $v_{j, t}^{i}$ is the closest node to point $p_{j, t}^{i}$.

4: We build an Euclidean spanning tree, denoted as $\operatorname{EST}\left(P_{j}^{i}\right)$, connecting points in $P_{j}^{i}$, as in $[10,11]$.

5: $\quad$ For each link $u v$ in the tree $\operatorname{EST}\left(P_{j}^{i}\right)$, assume that $u$ and $v$ are inside squarelet $\left(l_{u}^{1}, l_{u}^{2}\right)$ and squarelet $\left(l_{v}^{1}, l_{v}^{2}\right)$ respectively. Find a point $w$ in squarelet $\left(l_{v}^{1}, l_{u}^{2}\right)$ (or squarelet $\left.\left(l_{u}^{1}, l_{v}^{2}\right)\right)$, i.e., $u w v$ is a Manhattan path connecting $u$ and $v$. The resulted structure by uniting all such paths for all links in $\operatorname{EST}\left(P_{j}^{i}\right)$ will serve the routing guideline for multicast.

6: For each edge $u w$ in $\operatorname{EST}\left(P_{j}^{i}\right)$, find a node in each of the squarelets that are crossed by line $u w$. We connect these nodes in sequence to form a path, denoted as $\mathbf{P}(u, v)$, connecting points $u$ and $v$. Notice that here such structure may not be a tree. If this is the case, we could remove the cycles that do not contain nodes from $P_{j}^{i}$. Denote the resulting tree as $M T\left(P_{j}^{i}\right)$.

7: $\quad$ For each receiver $v_{l^{1}, l^{2}}$, if it is not inside the squarelet $\mathbf{s}$ containing point $p_{l^{1}, l^{2}}$, let $v_{l^{1}, l^{2}}^{\prime}$ be the node selected inside the squarelet $\mathbf{s}$. Notice that, such $v_{l^{1}, l^{2}}^{\prime}$ exists for every squarelets, with probability at least $1-2 / n$. Node $v_{l^{1}, l^{2}}^{\prime}$ then relay the data to node $v_{l^{1}, l^{2}}$ (the relay takes at most 2 hops). The final tree (including these additional relays) are called multicast tree $\operatorname{MTR}\left(U_{j}^{i}\right)$.

8: end for

9: The final tree (forest) consists of $\operatorname{MTR}\left(U_{j}^{i}\right)$ is the multicast tree (forest) $M T R\left(U_{j}\right)$.
}

Under this case, from Lemma 5, the length of multicast tree (forest) $T$ spanning all $k$ receivers is at most $\frac{3 c_{1}}{2} \frac{a}{\sqrt{m}} \cdot s$ with probability $1-\frac{4 c_{2}}{c_{1}^{2} \cdot s} \rightarrow 1$ when $s$ goes to $\infty$, here $c_{1}$ and $c_{2}$ are constants and $s$ is the number of subregions which contain at least one of $k$ receivers. Thus, from Lemma 3, we have

$$
L \leq \frac{3 c_{1}}{2} \cdot\left(\frac{a}{\sqrt{m}}\right) \cdot c_{7} \cdot k=\rho_{1} \cdot \frac{a}{\sqrt{m}} \cdot k
$$

with probability $\left(1-\frac{4 c_{2}}{c_{1}^{2} \cdot s}\right)\left(1-e^{-\frac{k^{2}}{8 m}}\right) \rightarrow 1$ when $s \rightarrow \infty$ and $\frac{k^{2}}{m} \rightarrow \infty$, where $\rho_{1}=\frac{3 c_{1} \cdot c_{8}}{2}$.

Clearly, the area of $D(\stackrel{2}{T})$, denoted by $|D(T)|$, is at most

$|D(T)| \leq 2 r \cdot L+k \cdot \pi r^{2} / 2 \leq 2 \rho_{1} \cdot \frac{a}{\sqrt{m}} \cdot k \cdot r+\frac{\theta_{1} \cdot \pi a^{2}}{2}$

from $k \leq \theta_{1} \frac{a^{2}}{r^{2}}$.

Before we present the lower bound capacity, we show the upper bound of the number of nodes that will receive a copy of data first.

LEMMA 19. Given a multicast tree (forest) constructed by Algorithm 1, the number of nodes that will get a copy of multicast data is, with high probability, at most $4 \rho_{1} \cdot \frac{k}{\sqrt{m}} \cdot \frac{r}{a} \cdot n+\theta_{1} \cdot \pi \cdot n$, when $k=O(m), \frac{k}{\sqrt{m}} \rightarrow \infty$ and $m=o\left(\frac{a^{2}}{r^{2}}\right)$, here $\rho_{1}$ is a constant.

Proof. Consider a set of receivers $U_{j}$ for the source node $v_{j}$. Let $T$ be the multicast tree (forest) $M T\left(P_{j}\right)$ constructed by Algorithm 1 . Let $X_{i}$ be the indicator variable denoting whether the $i$ th node $v_{i}$ will fall inside the region $D(T)$ for a multicast tree (forest) $T$. Clearly, $\operatorname{Pr}\left(X_{i}=1\right)=\frac{|D(T)|}{a^{2}}$. Notice that the area of $D(T)$, denoted by $|D(T)|$, is at most $2 \rho_{1} \cdot \frac{a}{\sqrt{m}} \cdot k \cdot r+k \cdot \pi r^{2} / 2$.

Assume $X$ is the number of nodes falling inside the region of $D(T)$. Clearly, $X=\sum_{i=1}^{n} X_{i}$ and $X$ is binomial distribution. By the Hoeffding's inequality, we have

$$
\begin{aligned}
\operatorname{Pr}(C & \left.>|D(T)| \cdot \frac{2 n}{a^{2}}\right) \leq \frac{|D(T)| \cdot \frac{2 n}{a^{2}} \cdot\left(1-\frac{|D(T)|}{a^{2}}\right)}{\left(|D(T)| \cdot \frac{2 n}{a^{2}}-\frac{n \cdot|D(T)|}{a^{2}}\right)^{2}} \\
& \leq \frac{2 a^{2}}{n \cdot|D(T)|} \leq \frac{2 c_{0} \sqrt{c}}{c_{2}} \cdot \frac{\sqrt{m}}{k} \frac{1}{\sqrt{n \cdot \log n}}
\end{aligned}
$$

which goes to 0 when $n \rightarrow \infty$ and $\frac{k}{\sqrt{m}} \rightarrow \infty$.

The last inequality comes from the assumption that $\frac{a}{r} \leq \sqrt{\frac{c \cdot n}{\log n}}$ from Theorem 2. Consequently, the number of nodes that can get a copy of the data for multicast within nodes $P_{i}$,w.h.p., is at most

$$
|D(T)| \cdot \frac{2 n}{a^{2}} \leq 4 \rho_{1} \cdot \frac{k}{\sqrt{m}} \cdot \frac{r}{a} \cdot n+\theta_{1} \cdot \pi \cdot n
$$

This finishes the proof.

Recall that, we can guarantee that each node will be able to transmit once every $\Delta$ time-slots. This implies that the total bits/sec achieved by all nodes is at least $n \cdot W / \Delta$. Consequently, the multicast capacity is at least

$$
\begin{array}{r}
\frac{n \cdot W / \Delta}{4 \rho_{1} \cdot \frac{k}{\sqrt{m}} \cdot \frac{r}{a} \cdot n+\theta_{1} \cdot \pi \cdot n}=\frac{W \cdot a \cdot \sqrt{m}}{4 \rho_{1} \Delta k r+\Delta \theta_{1} \pi \sqrt{m} a} \\
\geq \frac{W \cdot a \cdot \sqrt{m}}{4 \rho_{1} \Delta c_{9} \sqrt{m} a+\Delta \theta_{1} \pi \sqrt{m} a}=\frac{W}{4 \rho_{1} \Delta c_{9}+\Delta \theta_{1} \pi}
\end{array}
$$

The last inequality comes from Lemma 20.

LEMMA 20. When $k=O(m)$ and $\frac{k}{\sqrt{m}} \rightarrow \infty$, we have $k \cdot r \leq$ $c_{9} \sqrt{m} \cdot a$. 
PROOF. According to our assumption at the beginning $\sqrt{m} \leq$ $\frac{a}{r}$. In addition $\frac{k}{m} \leq \theta_{2}$, here $\theta_{2}$ is a constant. So we have

$$
\frac{k}{m} \leq \theta_{2} \leq \frac{\frac{a}{r}}{\sqrt{m}} \cdot \theta_{2}
$$

Thus, $\frac{k}{\sqrt{m}} \leq \theta_{2} \frac{a}{r}$. Hence, $k \cdot r \leq \theta_{2} \sqrt{m} \cdot a$, then the lemma follows by setting $c_{9}=\theta_{2}$.

THEOREM 21. The total multicast capacity $\Lambda(n)$ achievable by all multicast flows is at least $\rho_{3} \cdot W$, when $k=O(m), \frac{k}{\sqrt{m}} \rightarrow \infty$, $m=o\left(\frac{a^{2}}{r^{2}}\right)$. Here $\rho_{3}=\frac{1}{4 \rho_{1} \Delta c_{9}+\Delta \theta_{1} \pi}$.

Observed that the correctness of Theorem 21 relies on the fact that $\frac{a}{r} \leq \sqrt{\frac{c n}{\log n}}$ and $k \leq \theta_{1} \frac{a^{2}}{r^{2}}$. Thus, by letting $\frac{a}{r}=\sqrt{\frac{c n}{\log n}}$ for some small constant $c$, and $\rho_{4}=\tau_{3} \sqrt{c}$, based on Theorem 21, we have

COROLLARY 22. The multicast capacity for a hybrid wireless network $N_{n, m, a}$, when $k=O(m), \frac{k}{\sqrt{m}} \rightarrow \infty, m=o\left(\frac{a^{2}}{r^{2}}\right)$ and $a / r \leq \sqrt{\frac{c n}{\log n}}$, is at least

$$
\rho_{4} \cdot W
$$

Thus, the multicast capacity per flow (with $n$ sources) is at least

$$
\lambda_{k}(n)=\frac{\Lambda_{k}(n)}{n}=\Omega\left(\frac{W}{n}\right)
$$

Remember the above result is based on the pure hybrid routing, actually we can get tighter lower bound which is $\Omega\left(\frac{\sqrt{n}}{\sqrt{\log n} \cdot \sqrt{k}} \cdot W\right)$ (Results in [10]) for multicast capacity when we use the pure ad hoc routing strategy. This is because when the number of receivers for each source node is strictly less than the number of base stations, to constrict all wireless nodes to transfer data to their closest base stations first will not improve the capacity of the hybrid wireless network, even degrade the capacity.

\subsection{When $k=O\left(a^{2} / r^{2}\right)$ and $k=\Omega(m), \frac{m}{k} \rightarrow 0$}

Assume for any subregion $S_{i}$, the number of receivers existing in $S_{i}$ is $k_{i}$, then we know the expected value of $k_{i}, E\left(k_{i}\right)=\frac{k}{m} \rightarrow \infty$ when $\frac{m}{k} \rightarrow 0$.

LEMMA 23. The total length of the multicast tree (forest) $M T\left(P_{i}\right)$ spanning all $k$ receivers among all $m$ subregions is at most $\tau_{1} \cdot \sqrt{k}$. $a$ w.h.p., when $k=O\left(a^{2} / r^{2}\right), k=\Omega(m)$ and $\frac{m}{k} \rightarrow 0$, here $\tau_{1}$ is a constant depending only on $\theta_{1}$.

PROOF. According to the result of [11], we know that the total length $L$ of spanning tree (forest) $T$ is,

$$
L \leq \sum_{i=1}^{m}\left(4 \sqrt{2}+\frac{2 \sqrt{10}}{5} \cdot \sqrt{\theta_{1}}\right) \cdot \sqrt{k_{i}} \cdot \frac{a}{\sqrt{m}}
$$

Clearly, $L$ will get to its upper bound when $k_{i}=\frac{k}{m}$ for every $i$, $1 \leq i \leq m$. Thus,

$$
L \leq\left(4 \sqrt{2}+\frac{2 \sqrt{10}}{5} \cdot \sqrt{\theta_{1}}\right) \cdot \sqrt{k} \cdot a
$$

The theorem follows by letting $\tau_{1}=\left(4 \sqrt{2}+\frac{2 \sqrt{10}}{5} \cdot \sqrt{\theta_{1}}\right)$.

LEMMA 24. Given a multicast tree (forest) constructed by Algorithm 1, the number of nodes that will get a copy of multicast data is, with high probability, at most $\tau_{2} \frac{r \cdot \sqrt{k} \cdot n}{a}$, when $k \leq \theta_{1} \frac{a^{2}}{r^{2}}$ and $\frac{m}{k} \rightarrow 0$, here $\tau_{2}$ is a constant depending only on $\theta_{1}$.
PROOF. Consider a set of receivers $U_{1}$ for the source node $v_{1}$. Let $T$ be the multicast tree (forest) $M T\left(P_{i}\right)$ constructed by Algorithm 1 . Let $X_{i}$ be the indicator variable denoting whether the $i$ th node $v_{i}$ will fall inside the region $D(T)$ for a multicast tree (forest) $T$. Clearly, $\operatorname{Pr}\left(X_{i}=1\right)=\frac{|D(T)|}{a^{2}}$. Notice that the area of $D(T)$, denoted by $|D(T)|$, is at most

$$
|D(T)| \leq 2 r \cdot L+k \cdot \pi r^{2} / 2 \leq\left(2 \tau_{1}+\frac{\pi}{2} \sqrt{\theta_{1}}\right) \cdot r \cdot \sqrt{k} \cdot a
$$

from Lemma 23 and $k \leq \theta_{1} \frac{a^{2}}{r^{2}}$.

Assume $X$ is the number of nodes falling inside the region of $D(T)$. Clearly, $X=\sum_{i=1}^{n} X_{i}$ and $X$ is binomial distribution. From Hoeffding's inequality, and using the similar prove in Lemma 13 , we have

$$
\operatorname{Pr}\left(C \leq|D(T)| \cdot \frac{2 n}{a^{2}}\right) \geq 1-2 c_{7} \sqrt{c} \frac{1}{\sqrt{n \cdot \log n \cdot k}}
$$

which goes to 1 when $n$ goes to $\infty$. Here, $c$ and $c_{7}$ are constants. Thus the number of nodes that can get a copy of the data for multicast within nodes $P_{i}$,w.h.p., is at most

$$
|D(T)| \cdot \frac{2 n}{a^{2}} \leq\left(4 \tau_{1}+\pi \sqrt{\theta_{1}}\right) \cdot \frac{r}{a} \cdot \sqrt{k} \cdot n
$$

Setting $\tau_{2}=4 \tau_{1}+\pi \sqrt{\theta_{1}}$ finishes the proof.

Remember that we can guarantee that each node will be able to transmit once every $\Delta$ time-slots. This implies that the total bits/sec achieved by all nodes is at least $n \cdot W / \Delta$. Thus, the multicast capacity is at least

$$
\frac{n \cdot W / \Delta}{\tau_{2} \cdot \frac{r}{a} \cdot \sqrt{k} \cdot n}=\frac{1}{\tau_{2} \cdot \Delta} \frac{a}{r} \frac{W}{\sqrt{k}}
$$

By setting $\tau_{3}=\frac{1}{\tau_{2} \cdot \Delta}$, we have the following theorem.

THEOREM 25. The total multicast capacity $\Lambda(n)$ achievable by all multicast flows is at least $\tau_{3} \frac{a}{r} \frac{W}{\sqrt{k}}$, when $k \leq \theta_{1} \frac{a^{2}}{r^{2}}$ and $\frac{m}{k} \rightarrow 0$ and $a / r \leq \sqrt{\frac{c n}{\log n}}$ for some constants $c$ and $\tau_{3}$.

Based on Theorem 25, we have

COROLLARY 26. The multicast capacity for a random network of $n$ nodes and $m$ base regularly distributed base stations, when $k \leq \theta_{1} \frac{a^{2}}{r^{2}}$ and $\frac{m}{k} \rightarrow 0$ and $a / r \leq \sqrt{\frac{c n}{\log n}}$, is at least

$$
\left.\Lambda_{k}^{(} n\right) \geq \tau_{4} \frac{\sqrt{n}}{\sqrt{\log n} \cdot \sqrt{k}} \cdot W=\Omega\left(\frac{\sqrt{n}}{\sqrt{\log n} \cdot \sqrt{k}} \cdot W\right)
$$

The multicast capacity per flow (with $n$ sources) is at least

$$
\lambda_{k}(n)=\frac{\Lambda_{k}(n)}{n}=\Omega\left(\frac{1}{\sqrt{n \cdot \log n} \cdot \sqrt{k}} \cdot W\right)
$$

Under this case, our lower bound unifies the lower bound in [11], which means that when the number of receivers for each source node is strictly bigger than the number of base stations, two routing strategies give us same lower capacity bound for hybrid wireless network. Unfortunately, under the pure hybrid routing strategy, all source nodes existing in some subregion $S_{i}$ will send data to the base station $z_{i}$ when some receiver node(s) falling outside of $S_{i}$. Thus, the base station will become the bottleneck of the network when the number of source nodes exceeds some value. Assume every node is the source node, then the expected (source) 
nodes falling into subregion $S_{i}$ is $\frac{n}{m}$, so the total expected capacity achieved in $S_{i}$ is

$$
\frac{n}{m} \cdot \frac{1}{\sqrt{n \cdot \log n} \cdot \sqrt{k}} \cdot W
$$

from corollary 26 . On the other hand, the data rate for any base station to receiver from adjacent wireless node is also $W$. Therefore base station $z_{i}$ will become a bottleneck when $\frac{n}{m} \cdot \frac{1}{\sqrt{n \cdot \log n} \cdot \sqrt{k}}$. $W>W$.i.e.,

$$
\sqrt{\frac{n}{\log n}}>m \cdot \sqrt{k}
$$

Thus, when the above equation satisfies, we will turn to pure ad hoc routing strategy rather than pure hybrid routing strategy to avoid the bottleneck and get the same lower bound capacity.

\subsection{When $k=O(\sqrt{m})$}

By Lemma 15, there are total $k$ subregions which contain exactly one receiver inside w.h.p. under this case. Now, we show that the total length of multicast tree (forest) $T_{j}$ which spans all receivers of source node $v_{j}$ has upper bound by the following lemma.

LEMma 27. For each source node $v_{j}$, the total length of multicast tree (forest) $T_{j}$ which spans all $k$ receivers of $v_{j}$ is at most $c_{12} \cdot \frac{a \cdot k}{\sqrt{m}}$ with high probability when $k=O(\sqrt{m})$.

PROOF. According to the Lemma 16 and equation 3, we know the mean Euclidean distance between one pair of base station/receiver satisfies $\bar{L}<\frac{3}{2} E\left(L_{i}\right)=\frac{3 c_{3} \cdot a}{2 \sqrt{m}}$.

Assume, for source node $v_{j}$, the receiver existing in the subregion $S_{i}$ is $v_{i}$, we will construct a multicast structure for $U_{j}^{i}$ by Algorithm 1 where $U_{j}^{i}=\left\{v_{i}, z_{i}\right\}$. For simplicity, assume we pick up $w_{2}$ in the Algorithm 1, then the Euclidean distance $\left|u w_{2}\right|+\left|w_{2} v\right|$ is at most $\sqrt{2}|u v|$. Thus the number of squarelets crossed by line $u w_{2}$ and $w_{2} v$ is at most $\frac{\sqrt{2}|u v|}{\frac{r}{\sqrt{5}}}+1$. Then the length of resulting multicast tree $T_{j}^{i}$ spanning $v$ and $u$ satisfies

$$
\left|T_{j}^{i}\right| \leq\left(\frac{\sqrt{2}|u v|}{\frac{r}{\sqrt{5}}}+1\right) \frac{\sqrt{2} r}{\sqrt{5}}=2\left|v_{i} z_{i}\right|+\frac{\sqrt{2} r}{\sqrt{5}}
$$

Then we have the total length of tree (forest) $\left|T_{j}\right|$ satisfies

$$
\begin{array}{r}
\left|T_{j}\right|=\sum_{i=1}^{k}\left|T_{j}^{i}\right| \leq \sum_{i=1}^{k}\left(2\left|v_{i} z_{i}\right|+\frac{\sqrt{2} r}{\sqrt{5}}\right) \\
\leq \frac{3 c_{3} \cdot a \cdot k}{\sqrt{m}}+\frac{\sqrt{2} r}{\sqrt{5}} \cdot k \leq\left(3 c_{3}+\frac{\sqrt{2}}{\sqrt{5}}\right) \cdot \frac{a \cdot k}{\sqrt{m}}
\end{array}
$$

The last inequality comes from $\sqrt{m}<\frac{a}{r}$. This finishes the proof by letting $c_{12}=3 c_{3}+\frac{\sqrt{2}}{\sqrt{5}}$.

Notice the length of $T_{j}$ here satisfies the same condition as the case when $k=O\left(a^{2} / r^{2}\right), k=\Omega(m)$ and $\frac{m}{k} \rightarrow 0$. So we know that given a multicast tree (forest) $T_{j}$ constructed by Algorithm 1 , the number of nodes $C_{j}$ that will get a copy of multicast data from the source node $v_{j}$ is at most

$$
4 c_{12} \cdot \frac{k}{\sqrt{m}} \cdot \frac{r}{a} \cdot n+\theta_{1} \cdot \pi \cdot n
$$

w.h.p. from Lemma 19. Thus, we have

THEOREM 28. The multicast capacity for hybrid wireless network $N_{n, m, a}$ is at least $\Theta(W)$ when $k=O(\sqrt{m})$.
PROOF. Recall that, based on Algorithm 1, we can guarantee that each node will be able to transmit once every $\Delta$ time-slots. This implies that the total bits/sec achieved by all nodes is at least $n_{s} \cdot W / \Delta$. So, from $\sum_{j=1}^{n} \lambda_{j} \cdot C_{j} \geq \frac{n \cdot W}{\Delta}$, we have

$$
\left.\sum_{j=1}^{n} \lambda_{j} \geq \frac{n \cdot W}{\Delta\left(4 c_{12} \cdot \frac{k}{\sqrt{m}} \cdot \frac{r}{a} \cdot n+\theta_{1} \cdot \pi \cdot n\right.}\right)
$$

When the number of source node $n_{s}$ goes to $n$ and by Lemma 20, the theorem follows.

\subsection{When $k=\Omega\left(\frac{a^{2}}{r^{2}}\right)$}

In this case, we have proved that the upper bound on the total multicast capacity is only $\Theta(W)$. Obviously, the total multicast capacity for hybrid wireless network is at least the lower bound of the capacity for broadcast no matter we use either pure hybrid routing or pure ad hoc routing. In [9], Keshavarz-Haddad et al.. present a broadcast scheme to achieve capacity $\Theta(W)$. Thus, we have

THEOREM 29. The total multicast capacity $\Lambda_{k}(n)$ achievable by all multicast flows is at least $c_{7} W$, where $c_{7} \leq \frac{1}{\Delta+1}$ is a constant.

\section{LITERATURE REVIEW}

Gupta and Kumar [6] studied the asymptotic unicast capacity of a multi-hop wireless networks for both two different models. Under a non-interference protocol, when each wireless node is capable of transmitting at $W$ bits per second using a constant transmission range, the throughput achievable by each node for a randomly chosen destination is $\Theta\left(\frac{W}{\sqrt{n \log n}}\right)$ bits per second. Here $n$ in number of nodes. Furthermore, even the nodes are optimally assigned and transmission range is optimally chosen, the throughput is only $\Theta\left(\frac{W}{\sqrt{n}}\right)$ bits per second for each node.

Grossglauser and Tse recently showed that the unicast capacity can be improved by the mobility of wireless nodes regardless of delay. Their main result shows that the average long-term throughput per source-destination pair can be kept constant even when the number of nodes per unit area increases. Gastpar and Vetterli studied the capacity of random networks using relay in [1]. Chuah et al. [2] studied the capacity scaling in MIMO wireless systems under correlated fading. The capacity scaling in delay tolerant networks with heterogeneous mobile devices was studied by Garetto et al. [3]. Keshavarz-Haddad et al. studied the bounds for the capacity of wireless multihop networks imposed by topology and demand in [8]. Their techniques can be used to study unicast, multicast and broadcast capacity.

Broadcast capacity of both arbitrary networks and random networks has been studied in $[9,13]$. They showed that the broadcast capacity of a given network is $\Theta(W)$ for single source broadcast and the achievable broadcast capacity per flow is only $\Theta(W / n)$ if each of the $n$ nodes will serve as a source node. Keshavarz-Haddad et al. [4] studied the broadcast capacity with dynamic power adjustment for physical interference model.

Multicast capacity was also studied in the literature. Jacquet and Rodolakis [5] studied the scaling properties of multicast for random wireless networks. They claimed that the maximum rate at which a node can transmit multicast data is $O\left(\frac{W}{\sqrt{k n \log n}}\right)$. Recently, rigorous proofs of the multicast capacity were given in $[11,14]$. Li et al. [11] studied asymptotic multicast capacity for a large-scale random wireless networks. They showed the total multicast capacity is $\Theta\left(\sqrt{\frac{n}{\log n}} \cdot \frac{W}{\sqrt{k}}\right)$ when $k=O\left(\frac{n}{\log n}\right)$ and when $k=\Omega\left(\frac{n}{\log n}\right)$, 
the total multicast capacity is equal to the broadcast capacity, i.e., $\Theta(W)$. In addition, they used VC-theorem to show that the capacity achieved by the multicast scheme in [11] matches the asymptotic upper bounds with avoiding the bottleneck.

The capacity for hybrid wireless networks was not fully studied in the literature. Liu et al. [16] studied the unicast capacity of hybrid wireless network (a wireless ad hoc network with infrastructure). They essentially studied the unicast capacity of hybrid wireless networks under different network models. For the onedimensional network model, they showed that in a hybrid wireless network with $n$ ordinary nodes and $b$ base stations, the gain in capacity is substantial, increasing linearly with the number of base stations as long as $b \log b \leq n$. Based on the two-dimensional strip model, a hybrid wireless network requires a large number of base stations $b=\Omega(\sqrt{n})$ in order to obtain such a capacity increase. Kozat and Tassiulas [15] also studied the unicast capacity of ad hoc networks with a random flat topology under the present support of an infinite capacity infrastructure network. They showed that the per source node capacity of $\Theta(W / \log N)$ can be achieved in a random network scenario based on some assumptions, like the number of ad hoc nodes per access point (base station) is bounded, etc.

\section{CONCLUSIONS}

In this paper, we essentially studied the multicast capacity that can be achieved by hybrid networks with randomly distributed wireless nodes and regularly distributed base stations. We derived analytical upper bounds and lower bounds on multicast capacity of a wireless hybrid network based on different cases.

Observe that all our results are proved when the deployment region is a square with side-length $a$ and the transmission range of all nodes is uniform with value $r$. It is not difficult to show that all our results still apply when the deployment region is a fixed square with side length $a=1$, while the transmission range is selected appropriately, i.e., $r=\Theta\left(\sqrt{\frac{\log n}{c \cdot n}}\right)$ for some constant $c$. In addition, our results still hold when $r=1$ while the deployment region has a bounded aspect ratio such as a disk or a rectangular area when ratio width/height is bounded. Further, we considered the protocol interference model for random networks. The details of some computations are omitted here due to space limit.

There are some interesting questions left for study for multicast capacity of the hybrid networks. The first question is what is the multicast capacity when $m$ base stations randomly distributed. The second question is what is the multicast capacity when the link capacity is not uniform: shorter links will have larger capacity. The third question is what is the multicast capacity when the Gaussian channel is used, instead of assuming that each node has a fixed transmission range and has a fixed data rate $W$.

\section{REFERENCES}

[1] Gastpar, M., and Vetterli, M. On the capacity of wireless networks: the relay case. In IEEE INFOCOM (2002).

[2] Chuah, C.-N., Tse, D. N. C., Kahn, J. M., And VALENZUELA, R. A. Capacity scaling in mimo wireless systems under correlated fading. In IEEE Transactions On Information Theory (March 2002), vol. 48.

[3] Garetto, M., Giaccone, P., and Leonardi, E. Capacity scaling in delay tolerant networks with heterogeneous mobile nodes. In ACM MobiHoc (2007), pp. 41-50.
[4] Keshavarz-Haddad, A., And Riedi, R. On the broadcast capacity of multihop wireless networks: Interplay of power, density and interference. In IEEE (SECON) (2007).

[5] JacQuet, P., AND Rodolakis, G. Multicast scaling properties in massively dense ad hoc networks. In ICPADS (2005), pp. 93-99.

[6] Gupta, P., AND Kumar, P. Capacity of wireless networks. IEEE Transactions on Information Theory IT-46 (1999), 388-404.

[7] Grossglauser, M., And Tse, D. Mobility increases the capacity of ad hoc wireless networks. IEEE/ACM Transactions on Networking 10, 4 (2002), 477-486.

[8] Keshavarz-Haddad, A., AND Riedi, R. H. Bounds for the capacity of wireless multihop networks imposed by topology and demand. In ACM MobiHoc (2007), pp. 256-265.

[9] Keshavarz-Haddad, A., Ribeiro, V., And Riedi, R. Broadcast capacity in multihop wireless networks. In $A C M$ MobiCom ( 2006), pp. 239-250.

[10] LI, X.-Y., Multicast Capacity of Wireless Ad Hoc Networks, In IEEE/ACM Transaction on Networking (2008), to appear.

[11] LI, X.-Y., TANG, S.-J., AND OPHIR, F. Multicast capacity for large scale wireless ad hoc networks. In ACM Mobicom (2007).

[12] Penrose, M. The longest edge of the random minimal spanning tree. Annals of Applied Probability 7 (1997), 340-361.

[13] TAVLI, B. Broadcast capacity of wireless networks. IEEE Communication Letters 10, 2 (February 2006).

[14] Shakkottai S., LiU X. AND SRIKant R., The multicast capacity of ad hoc networks, Proc. ACM Mobihoc, 2007.

[15] Ulas C. Kozat and Leandros TAssiulas, Throughput capacity of random ad hoc networks with infrastructure support, Proc. ACM Mobihoc, 2003.

[16] Benyuan LiU, Patrick Thiran, Don Towsley, Capacity of a Wireless Ad Hoc Network with Infrastructure, Proc. ACM Mobihoc, 2007.

\section{APPENDIX}

Lemma 30 (ChebysheV's Inequality). For a variable $X$,

$$
\boldsymbol{P r}(|X-\mu| \geq A) \leq \frac{\operatorname{Var}(X)}{A^{2}},
$$

where $\mu=E(X), \operatorname{Var}(X)$ is the variance of $X$, and $A>0$.

Lemma 31 (HoEFFding'S INEQUALITY). Let $X_{1}, x_{2}, \cdots$, $X_{n}$ be independent random variables and $\operatorname{Pr}\left(X_{i} \in\left[a_{i}, b_{i}\right]\right)=1$. Let $S=\sum_{i}^{n} X_{i}$ and $E(S)$ be its expected value. Then, for $t>0$,

$$
\operatorname{Pr}(S-E(S) \geq n t) \leq \exp \left(-\frac{2 n^{2} t^{2}}{\sum_{i=1}^{n}\left(b_{i}-a_{i}\right)^{2}}\right)
$$

Lemma 32. Consider $n$ independent variables $X_{i} \in\{0,1\}$, $p=\operatorname{Pr}\left(X_{i}=1\right)$, and $X=\sum_{i=1}^{n} X_{i}$.

$$
\begin{aligned}
& \boldsymbol{P r}(X \leq \xi) \leq e^{\frac{-2(n \cdot p-\xi)^{2}}{n}}, \quad \text { when } 0<\xi \leq n \cdot p . \\
& \boldsymbol{P r}(X>\xi)<\frac{\xi(1-p)}{(\xi-n \cdot p)^{2}}, \quad \text { when } \xi>n \cdot p \text {. }
\end{aligned}
$$

